Discrete Comput Geom 34:11-24 (2005)

DOI: $10.1007 / \mathrm{s} 00454-005-1165-3$

\title{
On Levels in Arrangements of Curves, II: A Simple Inequality and Its Consequences*
}

\author{
Timothy M. Chan \\ School of Computer Science, University of Waterloo, \\ Waterloo, Ontario, Canada N2L 3G1 \\ tmchan@uwaterloo.ca
}

\begin{abstract}
We give a surprisingly short proof that in any planar arrangement of $n$ curves where each pair intersects at most a fixed number $(s)$ of times, the $k$-level has subquadratic $\left(O\left(n^{2-1 / 2 s}\right)\right)$ complexity. This answers one of the main open problems from the author's previous paper [Discrete Comput. Geom., 29:375-393, 2003], which provided a weaker upper bound for a restricted class of curves only (graphs of degree-s polynomials). When combined with existing tools (cutting curves, sampling, etc.), the new idea generates a slew of improved $k$-level results for most of the curve families studied earlier, including a near- $O\left(n^{3 / 2}\right)$ bound for parabolas.
\end{abstract}

\section{Introduction}

The $k$-Level Problem for Lines. We begin by re-examining an old result on a famous open problem in two-dimensional combinatorial geometry.

The problem, first investigated by Lovász [23] and Erdős et al. [18] in the early 1970s, is the following: for a set $P$ of $n$ points in the plane, consider the number of different subsets of size $k$ (called $k$-sets) that can be formed by intersecting $P$ with a halfplane. How big can this number be, asymptotically, as a function of $n$ and $k$ ?

For the special case $k=\lfloor n / 2\rfloor$, we are considering the number of different ways to bisect a point set into two equal halves with a line. In the dual the problem is known to be asymptotically equivalent [16] to bounding the worst-case complexity (number of vertices) of the $k$-level in an arrangement of $n$ lines, where the $k$-level is defined as the closure of all points that lie on exactly one line and strictly above exactly $k-1$ lines. Alternatively, as a "kinetic" problem in one dimension, we are seeking the maximum

\footnotetext{
* A preliminary version of this paper appeared in Proceeding of the 44th IEEE Symposium on the Foundations of Computer Science [11]. This work was supported in part by an NSERC Research Grant.
} 
number of changes that the $k$ th smallest point can undergo, for $n$ points moving linearly on the real line. These different views of the problem help explain its central place in combinatorial and computational geometry. In particular, the problem is related to the analysis of geometric algorithms for ham-sandwich cuts [22], range searching [13], geometric optimization with violations [9], and kinetic/parametric minimum spanning trees [3]. See the many books [16], [25], [26], [30] and surveys [5], [7], [20] for more background information.

In the discussion below, we focus on bounds that are functions of $n$ only, because as Agarwal et al. [1] showed, an $O(f(n))$ upper bound for this case automatically implies a " $k$-sensitive" $O((n / k) f(k))$ bound, for any well-behaved function $f$.

The initial papers by Lovász [23] and Erdős et al. [18] established an $O\left(n^{3 / 2}\right)$ upper bound and an $\Omega(n \log n)$ lower bound. The conjecture by Erdós et al. [18] was that the number of $k$-sets (or $k$-level vertices) is $o\left(n^{1+\varepsilon}\right.$ ) for any fixed constant $\varepsilon>0$. No progress on the upper bound was made for almost two decades until Pach et al. gave a complicated proof of an $O\left(n^{3 / 2} / \log ^{*} n\right)$ upper bound in 1992 [27]. No significant progress was made for several more years until Dey presented an elegant proof of an $O\left(n^{4 / 3}\right)$ upper bound in 1998 [15]. The lower bound meanwhile has been increased to $n 2^{\Omega(\sqrt{\log n})}$ by Tóth in 2001 [34]; this lower bound was known some time ago by Klawe, Paterson, and Pippenger [16] for the weaker case of "pseudo-lines" (see below).

Can Dey's $O\left(n^{4 / 3}\right)$ upper bound be improved? A positive answer seems difficult to establish with the existing approaches. One reason is that Dey's proof [15] —and most known $O\left(n^{3 / 2}\right)$ proofs (e.g., see the proceedings version of [1])—works not only for the $k$-set/ $k$-level problem, but for a more general problem (bounding the complexity of $n$ nonoverlapping concave chains in an arrangement). However, for this more general problem, there is a matching $\Omega\left(n^{4 / 3}\right)$ lower bound [17]. If a better upper bound exists, the proof is likely to be substantially different and tailor-made for the $k$-set $/ k$-level problem.

To renew hope for Erdôs et al.'s original conjecture, we suggest a new proof of the old $O\left(n^{3 / 2}\right)$ upper bound for the $k$-level problem. Our proof is quite different from all previous proofs and is very simple: roughly speaking, we give an inequality that relates the sizes of different levels around the $k$-level; from this inequality, a subquadratic bound for the $k$-level follows just by solving a recurrence.

The $k$-Level Problem for Curves. Unfortunately, we see no obvious ways to prove an $o\left(n^{3 / 2}\right)$ upper bound with the new idea, let alone an improvement to Dey's result. However, the idea adapts beautifully to a generalization of the problem-the $k$-level in an arrangement of curves. (In the one-dimensional kinetic setting, this corresponds to tracking changes to the $k$ th smallest point, where each point moves according to a nonlinear function in time.) This generalized problem is equally natural and fundamental, and has been studied intensively by several authors over the years [4], [10], [29], [33].

For example, our $O\left(n^{3 / 2}\right)$ proof works immediately for pseudo-lines ( $x$-monotone curves going from $x=-\infty$ to $x=\infty$, where each pair intersects at most once) and pseudo-segments ( $x$-monotone curve segments where each pair intersects at most once). Previous proofs also generalize to pseudo-lines, but their generalizations to arbitrary pseudo-segments require additional tools (to make pseudo-segments extendible [10]). As another feature of our proof, we can derive an $O\left(n^{3 / 2}+B\right)$ bound for the $k$-level in an arrangement of $n x$-monotone curve segments with $B$ "bad" pairs, where every good 
pair intersects at most once but every bad pair may intersect $O(1)$ times. To the author's knowledge, a similar bound for these "almost" pseudo-segment arrangements cannot be obtained easily (if at all) through previous approaches.

More importantly, our proof gives the first subquadratic $O\left(n^{2-1 / 2 s}\right)$ bound for general $x$-monotone curve segments (called $s$-intersecting curve segments) where each pair intersects at most $s$ times for a fixed constant $s$. Previously, a subquadratic bound was obtained in the predecessor of this paper [10] only when the curve segments are graphs of fixed-degree polynomials (or, slightly more generally, when repeated differentiation leads to a 1 - or 2-intersecting family). For degree-s polynomials, the previous bound was much worse- $\tilde{O}\left(n^{2-1 / 9 \cdot 2^{s-3}}\right)$ [10] (or $\tilde{O}\left(n^{2-1 / 6 \cdot 2^{s-3}}\right)$ using a recent improvement [24]). The notation $\tilde{O}$ hides polylogarithmic factors in this paper.

As Agarwal et al. [1] showed also for curves and curve segments, an $O(f(n))$ upper bound implies a $k$-sensitive $O((n / k) f(k) \beta(n / k))$ bound, for any well-behaved function $f$ and some slow-growing (inverse-Ackermann-like) function $\beta$.

The only major tool available for curves was pioneered by Tamaki and Tokuyama [33] and later improved and extended by Agarwal et al. [4] and in our previous paper [10]: the idea is to $c u t$ the curves into pseudo-segments, so that $k$-level results on these pseudosegments can be applied. Unfortunately, for odd $s$, it is generally not possible to cut $s$ intersecting curves into subquadratically many $(s-1)$-intersecting segments, as a simple example illustrates [10]. Our approach manages to obtain nontrivial $k$-level bounds directly, without cutting the curves.

This paper does not replace its predecessor [10], as bounds on the number of cuts turn out to have further applications, for example, in the combinatorial analysis of pseudoconcave chains [10], incidences [2], [4], and many faces [2], [4] in arrangements of curves, and parametric minimum spanning trees [10]. Our approach has no ramifications on these problems. This defect can be viewed as an advantage, if one believes that the $k$-level problem has lower complexity than these other problems.

While our approach does not require cutting, it can be combined with cutting to improve practically all existing $k$-level upper bounds for arrangements of curves other than pseudo-lines/segments. Table 1 summarizes the best previous results [4], [10] obtained without our new technique, and the results obtained using our new technique. (Bounds in both columns have been changed from the preliminary version of this paper [11], to take into account a newly improved cutting bound by Marcus and Tardos [24].)

Table 1. Upper bounds on the complexity of the $k$-level.

\begin{tabular}{lcc}
\hline \multicolumn{1}{c}{ Class of curves } & Previous result & New result \\
\hline Lines/pseudo-lines/pseudo-segments & $\tilde{O}\left(n^{4 / 3}\right)=O\left(n^{1.334}\right)$ & - \\
Parabolas/pseudo-parabolas & $\tilde{O}\left(n^{5 / 3}\right)=O\left(n^{1.667}\right)$ & $\tilde{O}\left(n^{3 / 2}\right)=\tilde{O}\left(n^{1.5}\right)$ \\
Pseudo-parabolic segments & $\tilde{O}\left(n^{16 / 9}\right)=O\left(n^{1.778}\right)$ & $O\left(n^{5 / 3}\right)=O\left(n^{1.667}\right)$ \\
Pairwise-intersecting pseudo-parabolas & $\tilde{O}\left(n^{14 / 9}\right)=O\left(n^{1.556}\right)$ & $O\left(n^{13 / 9}\right)=O\left(n^{1.445}\right)$ \\
Graphs of cubics & $\tilde{O}\left(n^{11 / 6}\right)=O\left(n^{1.834}\right)$ & $\tilde{O}\left(n^{5 / 3}\right)=O\left(n^{1.667}\right)$ \\
Graphs of quartics & $\tilde{O}\left(n^{23 / 12}\right)=O\left(n^{1.917}\right)$ & $\tilde{O}\left(n^{31 / 18}\right)=O\left(n^{1.723}\right)$ \\
Graphs of quintics & $\tilde{O}\left(n^{47 / 24}\right)=O\left(n^{1.959}\right)$ & $\tilde{O}\left(n^{161 / 90}\right)=O\left(n^{1.789}\right)$ \\
$s$-Intersecting curve segments (odd $s \geq 3)$ & $O\left(n^{2}\right)$ & $O\left(n^{2-1 / 2 s}\right)$ \\
$s$-Intersecting curve segments (even $s \geq 4)$ & $O\left(n^{2}\right)$ & $O\left(n^{2-1 / 2(s-1)}\right)$ \\
\hline
\end{tabular}


Perhaps the most notable of these results concerns the case of axis-aligned parabolas (which arises in tracking the $k$ th smallest distance for a set of linearly moving points). The history of the parabolic case illustrates the evolution of techniques well: Tamaki and Tokuyama's proof [33], which introduced the cutting approach, yielded an $O\left(n^{23 / 12}\right)=$ $O\left(n^{1.917}\right)$ bound; Dey's result [15] implied a reduction to $O\left(n^{17 / 9}\right)=O\left(n^{1.889}\right)$; in the previous paper [10], we utilized the cutting number more effectively and obtained an $\tilde{O}\left(n^{16 / 9}\right)=O\left(n^{1.778}\right)$ bound; Agarwal et al. [4] improved the cutting number itself and obtained a near- $O\left(n^{5 / 3}\right)$ bound (ignoring a super-polylogarithmic but small hidden factor); the present paper finds the best way to use the cutting number and finally brings the upper bound to near $O\left(n^{3 / 2}\right)$ (which, as the reader may recall, was the initial upper bound for the line case). The recent result by Marcus and Tardos [24] reduces the hidden factor somewhat and shows that the same bound carries over to pseudo-parabolas (2intersecting curves).

\section{The Proof for Lines}

In this section we illustrate our main idea for the case of lines. We give a new simple proof of an $O\left(n^{3 / 2}\right)$ upper bound for the size of the $k$-level. More generally, we actually show that any $O(i)$ consecutive levels have total size $O\left(n^{3 / 2} i^{1 / 2}\right)$.

For simplicity, we assume that the given arrangement is in general position.

Let $L_{k}$ denote the $k$-level. (By default, set $L_{k}$ to $y=-\infty$ if $k \leq 0$, or to $y=\infty$ if $k>n$.)

Let $t_{i}$ be the number of vertices in the arrangement strictly between $L_{k-i}$ and $L_{k+i}$.

Let $\Delta t_{i}=t_{i+1}-t_{i}$ be the number of vertices strictly between $L_{k-i-1}$ and $L_{k+i+1}$ that lie on $L_{k-i}$ or $L_{k+i}$. In other words, $\Delta t_{i}$ is the total number of vertices in $L_{k-i} \backslash L_{k-i-1}$ (i.e., "concave" vertices of $L_{k-i}$ ) and vertices in $L_{k+i} \backslash L_{k+i+1}$ (i.e., "convex" vertices of $\left.L_{k+i}\right)$.

Lemma 2.1. For an arrangement of lines,

$$
t_{i} \leq 2 i \Delta t_{i}+O\left(i^{2}\right)
$$

Proof. Take each vertex $u$ strictly between $L_{k-i}$ and $L_{k+i}$. Suppose $u$ is defined by the lines $\ell_{1}$ and $\ell_{2}$. For each $j \in\{1,2\}$, shoot a rightward ray from $u$ along $\ell_{j}$ until the ray hits $L_{k-i}$ or $L_{k+i}$ or reaches $x=\infty$; let $v_{j}$ be the point hit. If $v_{1}$ is to the left of $v_{2}$, charge $u$ to $v_{1}$; otherwise, charge $u$ to $v_{2}$. (See Fig. 1.)

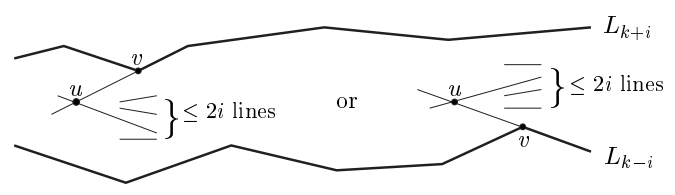

Fig. 1. The charging argument for Lemma 2.1 . 
Consider a point $v$ that receives charges. Then $v$ must be a concave vertex of $L_{k-i}$ or a convex vertex of $L_{k+i}$, or a point at $x=\infty$ strictly between $L_{k-i}$ and $L_{k+i}$; the total number of such points is at most $\Delta t_{i}+2 i-1$. For $v$ to receive a charge from $u$, the vertex $u$ must be defined by a line strictly between $L_{k-i}$ and $L_{k+i}$ at the $x$-coordinate of $v$; the number of such lines is at most $2 i$ (or, more carefully, at most $2 i-2$ ). Clearly, a line can define at most one vertex charged to $v$. Thus, each point $v$ receives at most $2 i$ charges.

We conclude that the total number of charges, $t_{i}$, is less than $2 i\left(\Delta t_{i}+2 i\right)$.

Theorem 2.2. For an arrangement of $n$ lines, the k-level has $O\left(n^{3 / 2}\right)$ vertices.

Proof. The inequality $t_{i} \leq 2 i\left(t_{i+1}-t_{i}\right)+O\left(i^{2}\right)$, i.e.,

$$
t_{i} \leq \frac{2 i}{2 i+1} t_{i+1}+O(i)
$$

forms a recurrence (or a difference equation, if equality holds). For the base case, we have $t_{n}=O\left(n^{2}\right)$. As shown in the Appendix (Lemma A.1), this recurrence solves to $t_{i}=O\left(n^{3 / 2} i^{1 / 2}\right)$. The size of the $k$-level is upper-bounded by $t_{2}$, so the theorem follows.

\section{Remarks.}

- The $O\left(n^{3 / 2} i^{3 / 2}\right)$ bound on the complexity of $O(i)$ consecutive levels was first shown by Welzl [35]. Dey's proof [15] improved this to $O\left(n^{4 / 3} i^{2 / 3}\right)$.

- A few previous papers (e.g., [1], [8], and [21]) have also examined relationships among sizes of different levels, but the above inequality has not been observed before.

- Like previous proofs, our proof can directly yield a $k$-sensitive $O(n \sqrt{k})$ upper bound of the size of the $k$-level: by using the known fact [6], [19] that the combined complexity of the first $O(k)$ levels is $O(n k)$, we obtain a better base case $t_{k}=$ $O(n k)$; solving the recurrence (with details omitted) now yields $t_{i}=O(n \sqrt{k i})$ for $i \leq k$.

- We can also directly prove the above-mentioned fact that the combined complexity of the first $O(i)$ levels is $O(n i)$ : redefining $t_{i}$ to be the number of vertices strictly below $L_{i}$, we can modify the charging argument to show that

$$
t_{i} \leq i \Delta t_{i}+O\left(i^{2}\right),
$$

which implies that $t_{i}=O(n i)$ (by Lemma A.1, or by a simple telescoping argument).

- Interestingly, we can also adapt the proof to show that for a set of $n$ line segments, their lower envelope (1-level) has $O(n \log n)$ vertices: redefining $t_{i}$ again to be the number of vertices strictly below $L_{i}$, we have this time

$$
t_{i} \leq i \Delta t_{i}+O(n i),
$$

if the ray-shooting process is modified to stop at endpoints as well (see the next section for more details); this recurrence solves to $t_{i}=O(n i \log (n / i)$ ) (by Lemma A.1). 
Although the worst-case complexity of the lower envelope is actually $\Theta(n \alpha(n))$ [30] (where $\alpha$ denotes the inverse Ackermann function), the above proof is simple. Tagansky [31] also gave an $O(n \log n)$ proof involving a recurrence of a somewhat similar form, but his recurrence was obtained using probabilistic arguments.

\section{The Generalization to Curves}

The new proof extends easily to pseudo-lines and pseudo-segments. In this section we consider general curves and curve segments. The generalization of Lemma 2.1 is straightforward. We state the inequality in a form that is sensitive to the number of "bad" pairs; this form will be beneficial in later proofs.

Throughout the paper we assume that "curve segments" are $x$-monotone and each pair intersects at most $O(1)$ times (the latter condition is not required in the lemma below and is needed subsequently). Unbounded curves can be viewed as special cases of curve segments (with endpoints at $x= \pm \infty$ ).

Given two curve segments $\gamma_{1}$ and $\gamma_{2}$ that intersect at least $s+1$ times, an $s$-lens refers to the portion of $\gamma_{1} \cup \gamma_{2}$ between some $s+1$ consecutive intersection points of $\gamma_{1} \cap \gamma_{2}$.

Let $\Lambda_{i}^{(s)}$ be the collection of all $s$-lenses fully lying strictly between $L_{k-i}$ and $L_{k+i}$. Let $t_{i}$ and $\Delta t_{i}$ be defined as in the previous section.

Lemma 3.1 (Main Inequality).

$$
t_{i} \leq 2 s i \Delta t_{i}+O\left(n i+\left|\Lambda_{i}^{(s)}\right|\right) .
$$

Proof. Take each vertex $u$ strictly between $L_{k-i}$ and $L_{k+i}$. Suppose $u$ is defined by the curve segments $\gamma_{1}$ and $\gamma_{2}$. If $u$ is the leftmost point of some $s$-lens in $\Lambda_{i}^{(s)}$ formed by $\gamma_{1}$ and $\gamma_{2}$, charge $u$ to this $s$-lens. Otherwise, for each $j \in\{1,2\}$, walk rightward from $u$ along $\gamma_{j}$ until $L_{k-i}$ or $L_{k+i}$ or an endpoint is hit; let $v_{j}$ be the point hit. If $v_{1}$ is to the left of $v_{2}$, charge $u$ to $v_{1}$; otherwise, charge $u$ to $v_{2}$. (See Fig. 2.)

Consider a point $v$ that receives charges. Then $v$ must be a vertex in $L_{k-i} \backslash L_{k-i-1}$ or $L_{k+i} \backslash L_{k+i+1}$, or an endpoint, or a point of discontinuity in $L_{k-i}$ or $L_{k+i}$; the total number of such points is $\Delta t_{i}+O(n)$. For $v$ to receive a charge from $u$, the vertex $u$ must be defined by a curve segment strictly between $L_{k-i}$ and $L_{k+i}$ at the $x$-coordinate of $v$; the number of such segments is at most $2 i$. Each curve segment can define at most $s$ vertices charged to $v$, because if there were $s+1$ such vertices, the leftmost such vertex would be the leftmost point of an $s$-lens strictly between $L_{k-i}$ and $L_{k+i}$. Thus, each point $v$ receives at most $2 s i$ charges.

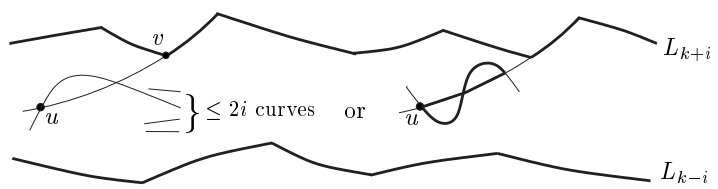

Fig. 2. The charging argument for Lemma 3.1, with $s=2$. (In the scenario to the left, $u$ is charged to a vertex $v \in L_{k+i} \backslash L_{k+i+1}$. In the scenario to the right, $u$ is charged to a 2-lens in $\Lambda_{i}^{(s)}$, shown in bold.) 
Each $s$-lens in $\Lambda_{i}^{(s)}$ receives just one charge. We conclude that the total number of charges, $t_{i}$, is at most $2 \operatorname{si}\left(\Delta t_{i}+O(n)\right)+\left|\Lambda_{i}^{(s)}\right|$.

Theorem 3.2. For an arrangement of s-intersecting curve segments, the $k$-level has $O\left(n^{2-1 / 2 s}\right)$ vertices. More generally, for an arrangement of $n$ curve segments where at most B pairs may intersect more than s times, the k-level has $O\left(n^{2-1 / 2 s}+B\right)$ vertices.

Proof. The first part follows from the second (with $B=0$ ).

For the second part, since $\left|\Lambda_{i}^{(s)}\right|=O(B)$ for all $i$, the recurrence is $t_{i} \leq 2 s \Delta t_{i}+$ $O(n i+B)$, with $t_{n}=O\left(n^{2}\right)$. By a change of variable $t_{i}^{\prime}:=t_{i}-c B$ for a sufficiently large constant $c$, the recurrence simplifies to $t_{i}^{\prime} \leq 2 s \Delta t_{i}^{\prime}+O(n i)$. As shown in the Appendix (Lemma A.1), this yields $t_{i}^{\prime}=O\left(n^{2-1 / 2 s} i^{1 / 2 s}\right)$, i.e., $t_{i}=O\left(n^{2-1 / 2 s}\right.$ $\left.i^{1 / 2 s}+B\right)$.

\section{Improvements by (Not) Cutting}

For pseudo-parabolas (2-intersecting curves going from $x=-\infty$ to $x=\infty$ ) or pseudoparabolic segments (2-intersecting curve segments), Theorem 3.2 implies an $O\left(n^{7 / 4}\right)$ bound. This bound can be improved further, by applying known techniques to $c u t$ the given curve segments (i.e., subdivide them by inserting new endpoints) into a pseudosegment family. The $k$-level is unchanged after the cuts. A natural first idea is to make the cuts and then apply the main inequality (with a larger $n$ but a smaller $s$ ). We discover, though, that it is possible - and in fact more effective- to avoid explicitly cutting the curve segments but instead directly estimate the number of "bad" lenses $\left|\Lambda_{i}^{(s)}\right|$ using these known techniques.

Given a class of curve segments, let $v^{(s)}(n)$ be the maximum size of a collection of nonoverlapping $s$-lenses in an arrangement of $n$ segments in the class. Here, a collection is nonoverlapping if each pair intersects only at a discrete set of points. The number of cuts required to turn $n$ curve segments into an $s$-intersecting family is clearly $\Omega\left(v^{(s)}(n)\right)$, but is also $O\left(v^{(s)}(n)\right)$ by the technique of Tamaki and Tokuyama [33] (see also [24]).

Lemma 4.1. $\left|\Lambda_{i}^{(s)}\right|=O\left(i^{2} v^{(s)}(n / i)\right)$.

Proof. Given a collection of $s$-lenses, its depth refers to the maximum number of $s$ lenses that a point can lie on. The key observation is that $\Lambda_{i}^{(s)}$ has depth $O(i)$ : if a point $v$ lies on an $s$-lens $\lambda \in \Lambda_{i}^{(s)}$, then $\lambda$ must be defined by a curve segment containing $v$ and another curve segment lying strictly between $L_{k-i}$ and $L_{k+i}$ at the $x$-coordinate of $v$.

As one step of their technique, Tamaki and Tokuyama [33] have basically shown (at least for $s=1$ ) that the maximum size of a collection of $s$-lenses of depth $O(i)$ is $O\left(i^{2} v^{(s)}(n / i)\right)$. For the sake of completeness, we quickly provide the proof, which employs random sampling in the style of Clarkson and Shor [14].

Pick a random subset $R$ of the given curve segments with $|R|=n / i$. Take each lens $\lambda \in \Lambda_{i}^{(s)}$ defined by curve segments $\gamma_{1}$ and $\gamma_{2}$. Put $\lambda$ in a subcollection $\Lambda(R)$ under the 
following conditions:

1. the curve segments $\gamma_{1}$ and $\gamma_{2}$ are in $R$, and

2. for every other curve segment $\gamma$, if $\gamma$ defines some lens in $\Lambda_{i}^{(s)}$ that contains an endpoint of $\lambda$ (i.e., the leftmost or rightmost point of $\lambda$ ), then $\gamma$ is not in $R$.

Then $\Lambda(R)$ is nonoverlapping, because if two lenses overlap, one lens must contain an endpoint of the other. Thus, $E[|\Lambda(R)|]=O\left(v^{(s)}(n / i)\right)$. On the other hand, because $\Lambda_{i}^{(s)}$ has depth $O(i)$, for a fixed lens $\lambda \in \Lambda_{i}^{(s)}$, we have $\operatorname{Pr}\{\lambda \in \Lambda(R)\} \approx(1 / i)^{2}(1-1 / i)^{O(i)}=$ $\Omega\left(1 / i^{2}\right)$. Thus, $E[|\Lambda(R)|]=\Omega\left(\left|\Lambda_{i}^{(s)}\right| / i^{2}\right)$. The lemma follows.

\section{Theorem 4.2.}

(a) For an arrangement of $n$ pseudo-parabolas, the $k$-level has $O\left(n^{3 / 2} \log ^{2} n\right)$ vertices.

(b) For an arrangement of $n$ extendible pseudo-parabolic segments [10] (i.e., 2-intersecting curve segments that can be extended to form 2-intersecting curves), the $k$-level has $O\left(n^{3 / 2} \log ^{2} n\right)$ vertices.

(c) For an arrangement of $n$ pseudo-parabolic segments, the k-level has $O\left(n^{5 / 3}\right)$ vertices.

(d) For an arrangement of $n$ s-intersecting curve segments for an even $s \geq 4$, the $k$-level has $O\left(n^{2-1 / 2(s-1)}\right)$ vertices.

Proof. (a) For pseudo-parabolas, the original paper by Tamaki and Tokuyama [33] established $v^{(1)}(n)=O\left(n^{5 / 3}\right)$. Agarwal et al. [4] improved this to $v^{(1)}(n)=O\left(n^{3 / 2}\right.$ $\left.(\log n)^{O\left(\alpha(n)^{2}\right)}\right)$ for axis-aligned parabolas and other special cases. The bound for general pseudo-parabolas was also improved to $v^{(1)}(n)=O\left(n^{8 / 5}\right)$ by Agarwal et al. [4] and Pinchasi and Radoičić [28], and later improved to $v^{(1)}(n)=O\left(n^{3 / 2} \log n\right)$ by Marcus and Tardos [24]. Thus, combining with Lemma 4.1, we can write the main inequality as

$$
t_{i} \leq 2 i \Delta t_{i}+O\left(n^{3 / 2} i^{1 / 2} \log (n / i)\right) .
$$

Solving the recurrence (e.g., by applying Lemma A.1 to $t_{i}^{\prime}:=t_{i} / \log n$ ) yields $t_{i}=$ $O\left(n^{3 / 2} i^{1 / 2} \log ^{2} n\right)$. (This result is also noted in Marcus and Tardos' paper [24].)

(b) For extendible pseudo-parabolic segments, we still have $v^{(1)}(n)=O\left(n^{3 / 2} \log n\right)$ by applying Marcus and Tardos' bound to the extensions of the segments. So, the same result follows.

(c) For general pseudo-parabolic segments, the previous paper [10, Theorem 7.2] showed that $v^{(1)}(n)=O\left(n^{5 / 3}\right)$ (by adapting Tamaki and Tokuyama's approach). The main inequality is now

$$
t_{i} \leq 2 i \Delta t_{i}+O\left(n^{5 / 3} i^{1 / 3}\right),
$$

which (by Lemma A.1) solves to $t_{i}=O\left(n^{5 / 3} i^{1 / 3}\right)$.

(d) For $s$-intersecting curve segments for even values of $s$, the previous paper [10, Remark after Lemma 7.1] showed that $v^{(s-1)}(n)=O\left(n^{2-1 /(s+1)}\right)$ (by modifying Tamaki and Tokuyama's approach). We thus have

$$
t_{i} \leq 2(s-1) i \Delta t_{i}+O\left(n^{2-1 /(s+1)} i^{1 /(s+1)}\right),
$$

which solves to $t_{i}=O\left(n^{2-1 / 2(s-1)} i^{1 / 2(s-1)}\right)$, since $2(s-1)>s+1$ for $s \geq 4$. 
Remarks. There is quite a large gap between the upper bounds in parts (b) and (c) in the above theorem. An open problem is to determine whether Marcus and Tardos' cutting bound would still hold for general pseudo-parabolic segments.

\section{Improvements by Bootstrapping}

For certain curve families, further improvements can be obtained by switching to a different inequality, stated below, which uses level bounds on smaller arrangements to obtain a better level bound overall.

Given a class of curve segments, let $\tau_{i}(n)$ be an upper bound on the number of vertices strictly between the $(k-i)$ - and $(k+i)$-level, over all arrangements of $n$ segments in the class and all $k$. Assume that $\tau_{i}$ is well behaved, i.e., $\tau_{i}(c n)=O\left(\tau_{i}(n)\right)$ for any fixed constant $c$.

Lemma 5.1. For an arrangement of $n$ curve segments and $i \leq j / 2$,

$$
t_{i}=O\left(\left(n+\frac{t_{j}}{j}\right) \frac{\tau_{i}(j)}{j}\right) .
$$

Proof. The proof follows an approach by Agarwal et al. [1] (originally used to derive a $k$-sensitive level bound for line segments).

Let $m \geq i$ be a parameter to be determined later. Divide the plane into $O\left(\left(n+\Delta t_{m}\right) / m\right)$ vertical slabs such that for each slab $\sigma$, the set $S_{\sigma}$ of all curve segments that define vertices of $L_{k-m} \backslash L_{k-m-1}$ and $L_{k+m} \backslash L_{k+m+1}$ and endpoints within $\sigma$ has size at most $m$. Define $T_{\sigma}$ to be the set of all curve segments that lie strictly between $L_{k-m}$ and $L_{k+m}$ at the left wall of $\sigma$; the size of $T_{\sigma}$ is at most $2 m$. Within the slab $\sigma$, each level strictly between $L_{k-m}$ and $L_{k+m}$ in the original arrangement is equal to a level in the arrangement of the subset $S_{\sigma} \cup T_{\sigma}$. Thus, $t_{i}$ is bounded by $\tau_{i}(3 m)$ times the number of slabs. We conclude that

$$
t_{i}=O\left(\left(n+\Delta t_{m}\right) \frac{\tau_{i}(m)}{m}\right) .
$$

To finish, just choose an index $m$ between $\lfloor j / 2\rfloor$ and $j-1$ such that $\Delta t_{m} \leq t_{j} /\lceil j / 2\rceil$. Such an index exists because $\sum_{m=\lfloor j / 2\rfloor}^{j-1} \Delta t_{m} \leq t_{j}$.

Lemma 5.1 is best applied after cutting the given segments first (resulting in a larger $n$ but a smaller $\tau_{i}$ function), as illustrated in the following example:

Theorem 5.2. For an arrangement of $n$ pseudo-parabolas where each pair intersects exactly twice, the $k$-level has $O\left(n^{13 / 9} \log ^{1 / 3} n\right)$ vertices.

Proof. Agarwal et al. [4] established the bound $v^{(1)}(n)=O\left(n^{4 / 3}\right)$ for this special kind of pseudo-parabolas. The main inequality combined with Lemma 4.1 becomes

$$
t_{i} \leq 2 i \Delta t_{i}+O\left(n^{4 / 3} i^{2 / 3}\right),
$$

which yields $t_{i}=O\left(n^{3 / 2} i^{1 / 2}\right)$ only. 
To obtain a better bound for small $i$, we cut the given arrangement into an arrangement of extendible pseudo-segments: by Agarwal et al.'s result [4], the curves can be cut into $O\left(n^{4 / 3}\right)$ pseudo-segments, and by the extendibility technique from our previous paper [10, Theorem 3.3], these can be further subdivided into $O\left(n^{4 / 3} \log n\right)$ extendible pseudo-segments. For extendible pseudo-segments, we have $\tau_{i}(j)=O\left(j^{4 / 3} i^{2 / 3}\right)$ by a generalization [32] of Dey's level bound [15]. Lemma 5.1 then gives

$$
t_{i}=O\left(\left(n^{4 / 3} \log n+\frac{n^{3 / 2}}{j^{1 / 2}}\right) j^{1 / 3} i^{2 / 3}\right) .
$$

Setting $j=\left\lfloor n^{1 / 3} / \log ^{2} n\right\rfloor$ yields $t_{i}=O\left(n^{13 / 9} i^{2 / 3} \log ^{1 / 3} n\right)$, for $i \leq j / 2$.

We now apply the same "bootstrapping" strategy to improve $k$-level bounds for graphs of univariate low-degree polynomial functions. For cubics, although Theorem 3.2 yields only an $O\left(n^{11 / 6}\right)$ bound and the best bound for cutting into pseudo-segments $\left(v^{(1)}(n)\right)$ is $\tilde{O}\left(n^{7 / 4}\right)$ [10], [24], we are able to obtain a $k$-level bound that is better than both, as shown in the theorem below.

In what follows, $F^{\prime}$ refers to the family of curve segments corresponding to the derivatives of the univariate functions whose graphs form the family $F$.

Theorem 5.3. For an arrangement of $n$ curve segments $F$ (e.g., graphs of cubics) such that $F^{\prime}$ is an extendible pseudo-parabolic family, the $k$-level has $O\left(n^{5 / 3} \log ^{8 / 3} n\right)$ vertices.

Proof. For such a family $F$, we have shown in the previous paper [10, Lemma 7.3] that $v^{(2)}(n)=O\left(n^{3 / 2}\right)$. The main inequality combined with Lemma 4.1 gives

$$
t_{i} \leq 4 i \Delta t_{i}+O\left(n^{3 / 2} i^{1 / 2}\right),
$$

which solves to $t_{i}=O\left(n^{7 / 4} i^{1 / 4}\right)$.

To refine the bound, we cut the arrangement into extendible pseudo-parabolic segments: by the differentiation technique from our previous paper [10, Observation 6.1], it suffices to cut $F^{\prime}$ into extendible pseudo-segments, and by Marcus and Tardos' cutting bound and our extendibility technique, we get $O\left(n^{3 / 2} \log ^{2} n\right)$ segments. For extendible pseudo-parabolic segments, we have $\tau_{i}(j)=O\left(j^{3 / 2} i^{1 / 2} \log ^{2} j\right)$ by (the proof of) Theorem 4.2(b). Therefore, Lemma 5.1 gives

$$
t_{i}=O\left(\left(n^{3 / 2} \log ^{2} n+\frac{n^{7 / 4}}{j^{3 / 4}}\right) j^{1 / 2} i^{1 / 2} \log ^{2} j\right) .
$$

Setting $j=\left\lfloor n^{1 / 3} / \log ^{8 / 3} n\right\rfloor$ yields $t_{i}=O\left(n^{5 / 3} i^{1 / 2} \log ^{8 / 3} n\right)$ for $i$ small.

Further bootstrapping steps can yield improved results for polynomials of any fixed degree $s$. To illustrate the strategy, we only give bounds for the $s=4$ and $s=5$ cases.

Theorem 5.4. For an arrangement of $n$ curve segments $F$ (e.g., graphs of quartics) such that $F^{\prime \prime}$ is an extendible pseudo-parabolic family, the k-level has $O\left(n^{31 / 18} \log ^{34 / 9} n\right)$ vertices. 
Proof. For such a family $F$, we have shown in the previous paper [10, Section 7] (by a repeated differentiation technique) that $v^{(3)}(n)=O\left(n^{3 / 2}\right)$ and $v^{(2)}(n)=O\left(n^{7 / 4}\right)$. The main inequality combined with Lemma 4.1 for $s=2$ gives

$$
t_{i} \leq 4 i \Delta t_{i}+O\left(n^{7 / 4} i^{1 / 4}\right),
$$

which solves to $t_{i}=O\left(n^{7 / 4} i^{1 / 4} \log (n / i)\right)$.

To refine the bound, we cut the arrangement so that $F^{\prime}$ becomes an extendible pseudoparabolic family: by the differentiation technique from our previous paper, it suffices to cut $F^{\prime \prime}$ into extendible pseudo-segments, and by Marcus and Tardos' cutting bound and our extendibility technique, we get $O\left(n^{3 / 2} \log ^{2} n\right)$ segments. For curve segments $F$ such that $F^{\prime}$ is an extendible pseudo-parabolic family, we have $\tau_{i}(j)=O\left(j^{5 / 3} i^{1 / 2} \log ^{8 / 3} j\right)$ by (the proof of) Theorem 5.3. Lemma 5.1 then gives

$$
t_{i}=O\left(\left(n^{3 / 2} \log ^{2} n+\frac{n^{7 / 4} \log n}{j^{3 / 4}}\right) j^{2 / 3} i^{1 / 2} \log ^{8 / 3} j\right) .
$$

Setting $j=\left\lfloor n^{1 / 3} / \log ^{4 / 3} n\right\rfloor$ yields $t_{i}=O\left(n^{31 / 18} i^{1 / 2} \log ^{34 / 9} n\right)$ for $i$ small.

Theorem 5.5. For an arrangement of $n$ curve segments $F$ (e.g., graphs of quintics) such that $F^{\prime \prime \prime}$ is an extendible pseudo-parabolic family, the k-level has $O\left(n^{161 / 90} \log ^{182 / 45} n\right)$ vertices.

Proof. For such a family $F$, we have shown in the previous paper (by the repeated differentiation technique) that $v^{(4)}(n)=O\left(n^{3 / 2}\right), v^{(3)}(n)=O\left(n^{7 / 4}\right)$, and $v^{(2)}(n)=$ $O\left(n^{15 / 8}\right)$. The main inequality combined with Lemma 4.1 for $s=3$ gives

$$
t_{i} \leq 6 i \Delta t_{i}+O\left(n^{7 / 4} i^{1 / 4}\right),
$$

which solves to $t_{i}=O\left(n^{11 / 6} i^{1 / 6}\right)$.

To refine the bound, we cut the arrangement so that $F^{\prime \prime}$ becomes an extendible pseudoparabolic family: by the differentiation technique from our previous paper, it suffices to cut $F^{\prime \prime \prime}$ into extendible pseudo-segments, and by Marcus and Tardos' cutting bound and our extendibility technique, we get $O\left(n^{3 / 2} \log ^{2} n\right)$ segments. For curve segments $F$ such that $F^{\prime \prime}$ is an extendible pseudo-parabolic family, we have $\tau_{i}(j)=O\left(j^{31 / 18} i^{1 / 2} \log ^{34 / 9} j\right)$ by Theorem 5.4. Lemma 5.1 then gives

$$
t_{i}=O\left(\left(n^{3 / 2} \log ^{2} n+\frac{n^{11 / 6}}{j^{5 / 6}}\right) j^{13 / 18} i^{1 / 2} \log ^{34 / 9} j\right) .
$$

Setting $j=\left\lfloor n^{2 / 5} / \log ^{12 / 5} n\right\rfloor$ yields $t_{i}=O\left(n^{161 / 90} i^{1 / 2} \log ^{182 / 45} n\right)$ for $i$ small.

\section{Additional Remarks}

Tóth's $n 2^{\Omega(\sqrt{\log n})}$ lower bound [34] remains the current record for the $k$-level problem, even for $s$-intersecting curves. At present, it is conceivable that Erdôs et al.'s $o\left(n^{1+\varepsilon}\right)$ conjecture [18] might hold for curves. 
We do not know whether the main idea here, of exploiting relationship among nearby levels, could help in resolving the original problem for lines in the plane. (For example, is it possible to reduce the coefficient 2 in Lemma 2.1 by increasing the overhead term?) An upcoming paper [12] demonstrates that the idea can be used for arrangements of surfaces in three dimensions, albeit with considerably more effort.

\section{Acknowledgments}

I thank a referee for comments that improved the presentation in this paper.

\section{Appendix. Solving the Recurrences}

Lemma A.1. Given constants $a>0$ and $b \geq 0$, the inequality

$$
t_{i} \leq a i \Delta t_{i}+O\left(n^{2-b} i^{b}\right)
$$

with $t_{n}=O\left(n^{2}\right)$ implies that

$$
t_{i}= \begin{cases}O\left(n^{2-1 / a} i^{1 / a}\right) & \text { if } 1 / a<b \\ O\left(n^{2-b} i^{b} \log (n / i)\right) & \text { if } 1 / a=b \\ O\left(n^{2-b} i^{b}\right) & \text { if } 1 / a>b\end{cases}
$$

Proof. The inequality can be rewritten as

$$
t_{i} \leq \frac{a i}{a i+1} t_{i+1}+O\left(n^{2-b} i^{b-1}\right) .
$$

Note that

$$
\prod_{j=i}^{m} \frac{a j}{a j+1}=O\left(e^{-\sum_{j=i}^{m}(1 /(a j+1))}\right)=O\left(e^{-(1 / a) \ln (m / i)}\right)=O\left(\left(\frac{i}{m}\right)^{1 / a}\right) .
$$

Expanding the recurrence reveals that

$t_{i}=O\left(t_{n}\left(\frac{i}{n}\right)^{1 / a}+\sum_{m=i}^{n} n^{2-b} m^{b-1}\left(\frac{i}{m}\right)^{1 / a}\right)=O\left(n^{2-1 / a} i^{1 / a}+n^{2-b} i^{1 / a} \sum_{m=i}^{n} m^{b-1 / a-1}\right)$.

The three cases follow, since

$$
\sum_{m=i}^{n} m^{b-1 / a-1}=\left\{\begin{array}{lll}
O\left(n^{b-1 / a}\right) & \text { if } & 1 / a<b \\
O(\log (n / i)) & \text { if } & 1 / a=b \\
O\left(i^{b-1 / a}\right) & \text { if } & 1 / a>b
\end{array}\right.
$$

\section{References}

1. P. K. Agarwal, B. Aronov, T. M. Chan, and M. Sharir. On levels in arrangements of lines, segments, planes, and triangles. Discrete Comput. Geom., 19:315-331, 1998. (Preliminary version in Proc. 13th ACM Sympos. Comput. Geom., pages 30-38, 1997.) 
2. P. K. Agarwal, B. Aronov, and M. Sharir. On the complexity of many faces in arrangements of circles. In Discrete and Computational Geometry: The Goodman-Pollack Festschrift (B. Aronov, S. Basu, J. Pach, M. Sharir, eds.), pages 1-24. Springer-Verlag, Berlin, 2003.

3. P. K. Agarwal, D. Eppstein, L. J. Guibas, and M. R. Henzinger. Parametric and kinetic minimum spanning trees. In Proc. 39th IEEE Sympos. Found. Comput. Sci., pages 596-605, 1998.

4. P. K. Agarwal, E. Nevo, J. Pach, R. Pinchasi, M. Sharir, and S. Smorodinsky. Lenses in arrangements of pseudo-circles and their applications. J. ACM, 51:139-186, 2004.

5. P. K. Agarwal and M. Sharir. Arrangements and their applications. In Handbook of Computational Geometry (J. Urrutia and J. Sack, eds.), pages 49-119. North-Holland, Amsterdam, 2000.

6. N. Alon and E. Győri. The number of small semispaces of a finite set of points in the plane. J. Combin. Theory Ser. A, 41:154-157, 1986.

7. A. Andrzejak. On $k$-Sets and Their Generalizations. Ph.D. thesis, Dept. of Computer Science, ETH Zürich, 2000.

8. T. M. Chan. Remarks on $k$-level algorithms in the plane. Manuscript, 1999.

9. T. M. Chan. Low-dimensional linear programming with violations. In Proc. 43rd IEEE Sympos. Found. Comput. Sci., pages 570-579, 2002.

10. T. M. Chan. On levels in arrangements of curves. Discrete Comput. Geom., 29:375-393, 2003.

11. T. M. Chan. On levels in arrangements of curves, II: a simple inequality and its consequences. In Proc. 44th IEEE Sympos. Found. Comput. Sci., pages 544-550, 2003.

12. T. M. Chan. On levels in arrangements of surfaces in three dimensions. In Proc. 16th ACM-SIAM Sympos. Discrete Algorithms, pages 232-240, 2005.

13. B. Chazelle and F. P. Preparata. Halfspace range search: an algorithmic application of $k$-sets. Discrete Comput. Geom., 1:3-93, 1986.

14. K. L. Clarkson and P. W. Shor. Applications of random sampling in computational geometry, II. Discrete Comput. Geom., 4:387-421, 1989.

15. T. K. Dey. Improved bounds on planar $k$-sets and related problems. Discrete Comput. Geom., 19:373-382, 1998.

16. H. Edelsbrunner. Algorithms in Combinatorial Geometry. Springer-Verlag, Berlin, 1987.

17. D. Eppstein. Geometric lower bounds for parametric matroid optimization. Discrete Comput. Geom., 20:463-476, 1998.

18. P. Erdős, L. Lovász, A. Simmons, and E. Straus. Dissection graphs of planar point sets. In A Survey of Combinatorial Theory (J. N. Srivastava, ed.), pages 139-154. North-Holland, Amsterdam, 1973.

19. J. E. Goodman and R. Pollack. On the number of $k$-subsets of a set of $n$ points in the plane. J. Combin. Theory Ser. A, 36:101-104, 1984.

20. D. Halperin. Arrangements. In Handbook of Discrete and Computational Geometry (J. E. Goodman and J. O'Rourke, eds.), 2nd edn., pages 529-562. CRC Press, Boca Raton, FL, 2004.

21. N. Katoh, H. Tamaki, and T. Tokuyama. Parametric polymatroid optimization and its geometric applications. Internat. J. Comput. Geom. Appl., 12:429-443, 2002.

22. C.-Y. Lo, J. Matoušek, and W. L. Steiger. Algorithms for ham-sandwich cuts. Discrete Comput. Geom., 11:433-452, 1994.

23. L. Lovász. On the number of halving lines. Ann. Univ. Sci. Budapest Eötvös Sect. Math., 14:107-108, 1971.

24. A. Marcus and G. Tardos. Intersection reverse sequences and geometric applications. In Proc. 12th Internat. Sympos. Graph Drawing, pages 349-359. Lecture Notes in Computer Science, vol. 3383. Springer-Verlag, Berlin, 2004.

25. J. Matoušek. Lectures on Discrete Geometry. Springer-Verlag, Berlin, 2002.

26. J. Pach and P. K. Agarwal. Combinatorial Geometry. Wiley-Interscience, New York, 1995.

27. J. Pach, W. Steiger, and E. Szemerédi. An upper bound on the number of planar $k$-sets. Discrete Comput. Geom., 7:109-123, 1992.

28. R. Pinchasi and R. Radoičić. Topological graphs with no self-intersecting cycle of length 4. In Proc. 19th ACM Sympos. Comput. Geom., pages 98-103, 2003.

29. M. Sharir. On $k$-sets in arrangements of curves and surfaces. Discrete Comput. Geom., 6:593-613, 1991.

30. M. Sharir and P. K. Agarwal. Davenport-Schinzel Sequences and Their Geometric Applications. Cambridge University Press, New York, 1995.

31. B. Tagansky. A new technique for analyzing substructures in arrangements of piecewise linear surfaces. Discrete Comput. Geom., 16:455-479, 1996. 
32. H. Tamaki and T. Tokuyama. A characterization of planar graphs by pseudo-line arrangements. In Proc. 8th Internat. Sympos. Algorithms and Computation, pages 123-132. Lecture Notes in Computer Science, vol. 1350. Springer-Verlag, Berlin, 1997.

33. H. Tamaki and T. Tokuyama. How to cut pseudoparabolas into segments. Discrete Comput. Geom., 19:265-290, 1998.

34. G. Tóth. Point sets with many k-sets. Discrete Comput. Geom., 26:187-194, 2001.

35. E. Welzl. More on $k$-sets of finite sets in the plane. Discrete Comput. Geom., 1:95-100, 1986.

Received December 31, 2003, and in revised form September 22, 2004, and November 10, 2004.

Online publication March 22, 2005. 